\title{
Neutral gas and the escape of ionizing radiation: Lessons from the low-redshift Green Peas
}

\author{
Anne Jaskot ${ }^{1,2}{ }^{\mathbb{D}}$, Jed McKinney ${ }^{2}$, Tara Dowd ${ }^{3}$, Sally Oey ${ }^{4}$, Min Yun ${ }^{2}$, \\ Claudia Scarlata ${ }^{5}$ and James Lowenthal ${ }^{6}$ \\ ${ }^{1}$ Astronomy Department, Williams College, Williamstown, MA 01267, USA \\ email: 08aej@williams.edu \\ ${ }^{2}$ Department of Astronomy, University of Massachusetts, Amherst, MA 01003, USA \\ ${ }^{3}$ The Chandra X-ray Center, Cambridge, MA 02138, USA \\ ${ }^{4}$ Department of Astronomy, University of Michigan, Ann Arbor, MI 48109, USA \\ ${ }^{5}$ Minnesota Institute for Astrophysics, University of Minnesota, Minneapolis, MN 55455, USA \\ ${ }^{6}$ Department of Astronomy, Smith College, Northampton, MA 01063, USA
}

\begin{abstract}
How galaxies reionized the universe remains an open question, but we can gain insights from the low-redshift Green Pea galaxies, one of the only known populations of Lyman continuum (LyC) emitters. Using VLA H I $21 \mathrm{~cm}$ observations and HST UV spectra of Green Peas, we investigate how neutral gas content and geometry influence LyC and Ly $\alpha$ escape. Our results suggest that LyC Emitters may have high ratios of star formation rate to $\mathrm{H}$ I mass. Low gas covering fractions are common among the population, but not all sightlines are optically thin. Based on the observed relationship between high ionization parameters, low metallicities, and narrow Ly $\alpha$ profiles, we propose that weak stellar feedback at low metallicities results in a gas geometry of dense clumps within a low-density medium, which facilitates Ly $\alpha$ and LyC escape. We address the implications of these results for identifying $\mathrm{LyC}$ emitters at high redshift with $J W S T$ and ALMA.
\end{abstract}

Keywords. Galaxies: evolution, Galaxies: starburst, Intergalactic medium, Galaxies: ISM, Radiative transfer, Stars: massive

\section{Introduction}

One of the main goals of JWST is to identify the galaxies that reionized the universe. We currently do not know which galaxies dominate reionization or how much ionizing Lyman continuum (LyC) radiation escapes from galaxies into the intergalactic medium (IGM; e.g., Robertson et al. 2015; Madau \& Haardt 2015; Finkelstein et al. 2019; Naidu et al. 2019). Because the high-redshift IGM absorbs LyC radiation, we cannot directly study LyC escape during the epoch of reionization. However, we can gain important insights by studying galaxies in the low-redshift universe and investigating the physical factors that affect the escape of ionizing radiation.

The "Green Pea" (GP) galaxies are one of the most valuable low-redshift samples for probing the mechanisms behind LyC escape. Discovered in the Sloan Digital Sky Survey (SDSS) at $z<0.4$ Cardamone et al. 2009, the GPs share a number of properties with $z>2$ galaxies, such as high [O III] $\lambda 5007$ equivalent widths (200-2000 $)$, low stellar masses $\left(M_{*} \sim 10^{7}-10^{10} \mathrm{M}_{\odot}\right)$, high specific star formation rates $\left(\mathrm{sSFRs}=10^{-9}-10^{-7} \mathrm{M}_{\odot} \mathrm{yr}^{-1}\right)$, and low metallicities $\left(\sim 0.2 \mathrm{Z}_{\odot}\right.$; e.g., Cardamone et al. 2009; Amorín et al. 2010; Izotov 
et al. 2011; 2017b; Nakajima \& Ouchi 2014; Smit et al. 2014; Hagen et al. 2016). Like high-redshift galaxies, the GPs show elevated ratios of [O III] $\lambda 5007 /[\mathrm{O}$ II] $\lambda 3727$, which indicates a highly ionized interstellar medium and which may be a diagnostic of LyC escape (e.g., Jaskot \& Oey 2013; Nakajima \& Ouchi 2014).

Indeed, the GPs are the only known population of low-redshift star-forming galaxies where LyC escape appears to be common. All eleven GPs probed so far show LyC escape, with escape fractions ranging from $2-72 \%$ (Izotov et al. 2016; Izotov et al. 2018). Likewise, LyC emission has been detected from $z \sim 3$ galaxies with strong [O III] emission (e.g., Vanzella et al. 2016; Fletcher et al. 2019), although the average LyC escape fraction of this population may be $<15 \%$ (Rutkowski et al. 2017; Naidu et al. 2018).

In this work, we present recent analyses of the GPs' neutral gas properties using Very Large Array (VLA) $21 \mathrm{~cm}$ observations and Hubble Space Telescope (HST) UV spectra (Jaskot et al. 2017; McKinney et al. 2019; Jaskot et al. 2019). We selected a sample of $17 \mathrm{GPs}$ with $[\mathrm{O} \mathrm{III}] /[\mathrm{O} \mathrm{II}]$ ratios $\geqslant 7$ from SDSS. These galaxies are the most highly ionized star-forming galaxies at low redshift, and one galaxy in the sample, $\mathrm{J} 160810+352809$ (J1608), has the highest [O III]/[O II] ratio among SDSS star-forming galaxies $([\mathrm{O} \mathrm{III}] /[\mathrm{O} \mathrm{II}]=34.9)$. We have obtained VLA $21 \mathrm{~cm}$ spectra of J1608, and all 17 GPs in the sample have far-UV spectra from the HST Cosmic Origins Spectrograph.

\section{H I $21 \mathrm{~cm}$ Results: High SFR/ $N_{\mathrm{HI}}$ in LyC Emitters?}

The total H I content of a galaxy represents the amount of raw material available to form molecular gas and stars. Observations show that $\mathrm{H}$ i to stellar mass ratios increase with specific star formation rate and decrease with stellar mass (e.g., Huang et al. 2012b). Based on empirical scaling relations between H I masses, galaxy structure, and galaxy color (e.g., Huang et al. 2012b), we would expect low-mass starbursts like the GPs to have high $\mathrm{H}$ I gas fractions, as abundant $\mathrm{H}$ i fuels their intense star formation.

Instead, we find a $3 \sigma$ upper limit on J1608's $\mathrm{HI}$ mass of $\log \left(M_{\mathrm{HI}} / \mathrm{M}_{\odot}\right)=8.14$ (McKinney et al. 2019). This H I mass is not unusual for a galaxy of J1608's stellar mass $\left(\log \left(M_{*} / \mathrm{M}_{\odot}\right)=7.04\right.$ (Izotov et al. 2017b). However, it is unusual given J1608's extremely high sSFR $\left(6.85 \times 10^{-7} \mathrm{M}_{\odot} \mathrm{yr}^{-1}\right)$ and falls below the predictions of all $\mathrm{H} \mathrm{I}$ scaling relations for low-mass galaxies (McKinney et al. 2019). In other words, J1608 has a surprisingly high ratio of SFR to H I mass compared with other low-redshift galaxies (Figure 1a). Interestingly, two other galaxies show similarly high $\mathrm{SFR} / M_{\mathrm{HI}}$ ratios: Tololo 1247-232 (Puschnig et al. 2017) and Haro 11 (Pardy et al. 2016), both of which are known LyC emitters. Like other GPs, J1608 has a young burst age (<3 Myr; Izotov et al. 2017b; Jaskot et al. 2017) and likely a high ionizing photon production rate (Schaerer et al. 2016; Izotov et al. 2017a). Consequently, the standard conversion from $\mathrm{H} \alpha$ luminosity to SFR may be unreliable for J1608, and Figure 1a may instead show that LyC-emitting galaxies have higher $\mathrm{H} \alpha$ luminosities and higher ionizing fluxes per unit $\mathrm{H}$ I mass.

\section{UV Spectra Results: Gas Geometry and Ly $\alpha$ and LyC Escape}

The Ly $\alpha$ emission line and UV metal absorption lines can give us alternative perspectives on the neutral gas in GPs. Both models and observations suggest that the Ly $\alpha$ spectral profile width is sensitive to the H I column density, where higher column densities lead to more Ly $\alpha$ scattering and broader Ly $\alpha$ emission profiles (e.g., Verhamme et al. 2015; Izotov et al. 2018). Most, but not all, of the 17 highly ionized GPs in our sample are Ly $\alpha$ emitters (Jaskot et al. 2017; Jaskot et al. 2019). Two of the GPs likely do not have LyC escape along our line of sight, as they show deep Ly $\alpha$ absorption indicative of high $\mathrm{H}$ I column densities. However, most of the sample has strong, narrow Ly $\alpha$ emission profiles, as narrow as confirmed LyC emitters and suggestive of low H i column densities. 

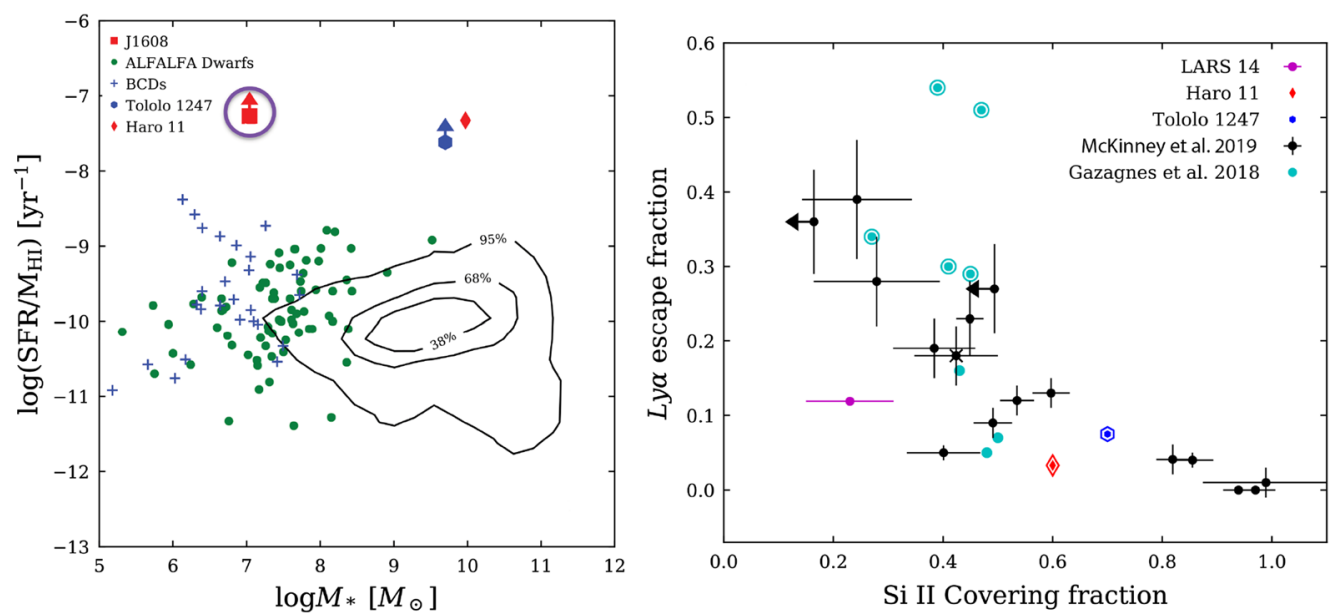

Figure 1. (a) Left: $\mathrm{SFR} / M_{\mathrm{HI}}$ vs. stellar mass. The red square inside the purple circle shows the limit for J1608. The blue hexagon and red diamond represent two confirmed LyC emitters (Puschnig et al. 2017; Pardy et al. 2016). Contours show SDSS galaxies with H I masses (Huang et al. 2012b). Green circles and blue crosses show dwarf galaxies (Huang et al. 2012a; Thuan et al. 2016). (b) Right: Ly $\alpha$ escape fraction correlates strongly with the low-ionization gas covering fraction for our GP sample (black points) and other GPs and LyC emitters (colored points). Figures adapted from McKinney et al. (2019).

Some of these Ly $\alpha$-emitting GPs, including J1608, show broad, underlying Ly $\alpha$ absorption in addition to their narrow Ly $\alpha$ emission. The derived $\mathrm{H}$ I column densities from the Ly $\alpha$ absorption components in the Ly $\alpha$-emitting and pure Ly $\alpha$-absorbing GPs range from $\log \left(N_{\mathrm{HI}} / \mathrm{cm}^{-2}\right)=19.5$ to 21.5 , several orders of magnitude too high for LyC escape and seemingly in contradiction to the narrow Ly $\alpha$ emission profiles (McKinney et al. 2019). These observations suggest that the neutral gas in the GPs is inhomogeneous, with optically thin sight lines coexisting with higher column density gas. From the GPs' UV metal absorption lines, we find further evidence for an inhomogeneous gas geometry. Their saturated line ratios and non-zero residual fluxes are consistent with partial gas covering fractions in the Ly $\alpha$-emitting GPs (McKinney et al. 2019).

We find that gas covering fraction is one of the physical properties that correlates most closely with Ly $\alpha$ escape fraction (Figure 1b; McKinney et al. 2019; Jaskot et al. 2019). This correlation suggests that the interstellar medium (ISM) porosity regulates $\mathrm{Ly} \alpha$ and $\mathrm{LyC}$ escape, with gaps within denser gas providing the avenues for Ly $\alpha$ and LyC photons to travel. However, these gaps cannot be true holes devoid of gas. If they were, Ly $\alpha$ photons would escape freely, without scattering, and would not form the double-peaked profiles we observe (Verhamme et al. 2015).

In addition to porosity, the ionization of the ISM may also affect Ly $\alpha$ and LyC escape. We examine the physical properties that correlate with Ly $\alpha$ profile width, specifically the separation of the blue and red Ly $\alpha$ emission peaks, $\Delta v_{\text {Ly } \alpha}$. A more highly ionized ISM, as traced by line ratios such as high $[\mathrm{O} \mathrm{III}] /[\mathrm{O} \mathrm{II}]$ and low $[\mathrm{OI}] \lambda 6300 / \mathrm{H} \beta$, is correlated with narrower Ly $\alpha$ profiles (Figure 2a; Jaskot et al. 2019). A more highly ionized ISM could be associated with lower $\mathrm{H}$ I column densities and hence narrow Ly $\alpha$ profiles. In this picture, the Ly $\alpha$ and LyC photons would travel through low, but not zero, column density gas channels between denser neutral clouds. Ionization would control the column density of these channels, which would then affect the Ly $\alpha$ profile widths.

Intriguingly, narrow $\Delta v_{\text {Ly } \alpha}$ also correlates strongly with low gas metallicities (Figure 2b; Jaskot et al. 2019). This trend cannot be explained by dust extinction, the 
(a)

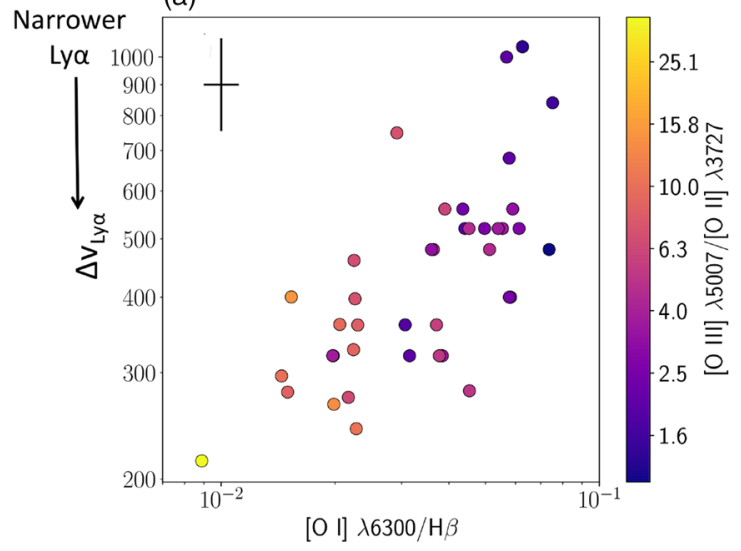

(b)

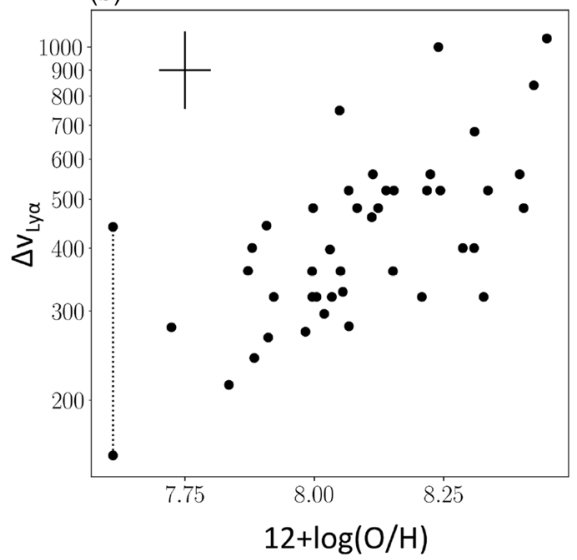

Figure 2. (a) The separation of the red and blue Ly $\alpha$ emission peaks, $\Delta v_{\mathrm{Ly} \alpha}$, in the GPs correlates with measures of ionization, such as [O III $\lambda 5007 /[\mathrm{O} I \mathrm{II}] \lambda 3727$ (color scale) and [O I] $\lambda 6300 / \mathrm{H} \beta$. (b) We observe an equally strong trend between $\Delta v_{\mathrm{Ly} \alpha}$ and metallicity. The dotted line connects two measures of $\Delta v_{\mathrm{Ly} \alpha}$ for a GP with a triple-peaked Ly $\alpha$ profile. Reproduced from Jaskot et al. (2019).

mass-metallicity relation, or metallicity-dependent ionizing luminosities, as none of these factors correlates strongly with $\Delta v_{\mathrm{Ly} \alpha}$. Rather, high ionization parameters appear to lie at the root of this trend, since high $[\mathrm{O}$ III $] /[\mathrm{O}$ II] ratios do correlate with low metallicities.

We propose a physical scenario that may explain the observed correlation between low metallicities, high ionization, and narrow Ly $\alpha$ profiles (Jaskot et al. 2019) and which may also explain why GPs so often show signs of LyC escape. At high metallicity, strong stellar winds push neutral gas into a relatively homogeneous superbubble shell, and LyC and Ly $\alpha$ photons encounter few optically thin pathways. Conversely, at low metallicity, mechanical stellar feedback is weaker (e.g., Vink et al. 2001; Ramachandran et al. 2019), and hence, low-metallicity clusters may retain more of their dense natal gas, particularly at young ages. This dense gas is likely clumpy; dense clumps near the ionizing source exhibit high ionization parameters, and low-density paths between the clumps allow Ly $\alpha$ and LyC photons to escape. This physical picture is consistent with the high-ionization emission, inhomogeneous geometries, young ages, and weak outflows observed in the GPs.

\section{Implications}

Highly ionized galaxies like the GPs frequently show direct or indirect evidence of LyC escape and may be similar to the galaxies that reionized the universe. At high redshift, JWST may identify candidate LyC emitters via high ionization line ratios, such as $[\mathrm{O}$ III $] /[\mathrm{O}$ II $]$ and $[\mathrm{O} \mathrm{I}] /[\mathrm{O}$ III] . In the case of ALMA, such galaxies will show high [O III $] \lambda 88 \mu \mathrm{m} /[\mathrm{C}$ II $] \lambda 158 \mu \mathrm{m}$, which appears to be a common property of galaxies in the epoch of reionization (Inoue et al. 2016; Carniani et al. 2017; Hashimoto et al. 2019).

LyC emitters may show high ratios of ionizing radiation to H I mass. However, these high ratios could arise from boosted ionizing photon production and do not necessarily mean that LyC-emitting galaxies are deficient in neutral gas. In fact, we find high neutral gas columns in LyC emitter candidates. ALMA could therefore potentially detect molecular gas in LyC emitters, although their low metallicities may make such detections challenging. The significant neutral gas columns in LyC-emitter candidates are consistent with weak mechanical feedback in low-metallicity starbursts at young ages. 
We have uncovered a possible link between high nebular ionization, low metallicities, and low H I column densities. Because low H I column densities lead to narrow Ly $\alpha$ spectral profiles, this link may help us constrain the Ly $\alpha$ spectral shape produced by highredshift galaxies before its subsequent radiative transfer through the IGM. Paradoxically, weak feedback at low metallicities may enhance the ISM porosity, generating a low-density inter-clump medium through which ionizing photons may escape. The inhomogeneity of the neutral gas implies that LyC escape will depend on orientation, and large samples will be required to pin down the dependence of LyC escape fraction on galaxy properties. To this end, we are undertaking the Low-Redshift Lyman Continuum survey (PI Jaskot), a Cycle 26 HST program, which will measure escape fractions for 66 galaxies and systematically test indicators of LyC escape.

\section{References}

Amorín, R. O., Pérez-Montero, E., \& Vílchez, J. M. 2010, ApJ (Letters), 715, L128

Cardamone, C., Schawinski, K., Sarzi, M., et al. 2009, MNRAS, 399, 1191

Carniani, S., Maiolino, R., Pallottini, A., et al. 2017, A\& A, 605, A42

Finkelstein, S. L., D'Aloisio, A., Paardekooper, J.-P., et al. 2019, ApJ, 879, 36

Fletcher, T. J., Tang, M., Robertson, B. E., et al. 2019, ApJ, 878, 87

Hagen, A., Zeimann, G. R., Behrens, C., et al. 2016, ApJ, 817, 79

Hashimoto, T., Inoue, A. K., Mawatari, K., et al. 2019, PASJ, 70

Huang, S., Haynes, M. P., Giovanelli, R., et al. 2012, AJ, 143, 133

Huang, S., Haynes, M. P., Giovanelli, R., et al. 2012, ApJ, 756, 113

Inoue, A. K., Tamura, Y., Matsuo, H., et al. 2016, Science, 352, 1559

Izotov, Y. I., Guseva, N. G., \& Thuan, T. X. 2011, ApJ, 728, 161

Izotov, Y. I., Schaerer, D., Thuan, T. X., et al. 2016, MNRAS, 461, 3683

Izotov, Y. I., Guseva, N. G., Fricke, K. J., et al. 2017, MNRAS, 467, 4118

Izotov, Y. I., Thuan, T. X., \& Guseva, N. G. 2017, MNRAS, 471, 548

Izotov, Y. I., Worseck, G., Schaerer, D., et al. 2018, MNRAS, 478, 4851

Jaskot, A. E. \& Oey, M. S. 2013, ApJ, 766, 91

Jaskot, A. E., Oey, M. S., Scarlata, C., et al. 2017, ApJ (Letters), 851, L9

Jaskot, A. E., Dowd, T., Oey, M. S., et al. 2019, ApJ, Submitted

Madau, P. \& Haardt, F. 2015, ApJ (Letters), 813, L8

McKinney, J. H., Jaskot, A. E., Oey, M. S., et al. 2019, ApJ, 874, 52

Naidu, R. P., Forrest, B., Oesch, P. A., et al. 2018, MNRAS, 478, 791

Naidu, R. P., Tacchella, S., Mason, C. A., et al. 2019, arXiv:1907.13130

Nakajima, K. \& Ouchi, M. 2014, MNRAS, 442, 900

Pardy, S. A., Cannon, J. M., Östlin, G., et al. 2016, AJ, 152, 178

Puschnig, J., Hayes, M., Östlin, G., et al. 2017, MNRAS, 469, 3252

Ramachandran, V., Hamann, W.-R., Oskinova, L. M., et al. 2019, A\&\&A, 625, A104

Robertson, B. E., Ellis, R. S., Furlanetto, S. R., et al. 2015, ApJ (Letters), 802, L19

Rutkowski, M. J., Scarlata, C., Henry, A., et al. 2017, ApJ (Letters), 841, L27

Schaerer, D., Izotov, Y. I., Verhamme, A., et al. 2016, A\&A (Letters), 591, L8

Smit, R., Bouwens, R. J., Labbé, I., et al. 2014, ApJ, 784, 58

Thuan, T. X., Goehring, K. M., Hibbard, J. E., et al. 2016, MNRAS, 463, 4268

Vanzella, E., de Barros, S., Vasei, K., et al. 2016, ApJ, 825, 41

Verhamme, A., Orlitová, I., Schaerer, D., et al. 2015, A\&A, 578, A7

Vink, J. S., de Koter, A., \& Lamers, H. J. G. L. M. 2001, A\&3A, 369, 574 